\title{
The G-Protein-Coupled Receptor SRX-97 Is Required for Concentration-Dependent Sensing of Benzaldehyde in Caenorhabditis elegans
}

\author{
Nagesh Y. Kadam, ${ }^{1}$ Sukanta Behera, ${ }^{1}$ Sandeep Kumar, $^{3}$ Anindya Ghosh-Roy, ${ }^{3}$ and ${ }^{-K a v i t a ~ B a b u}{ }^{1,2}$ \\ https://doi.org/10.1523/ENEURO.0011-20.2020 \\ ${ }^{1}$ Department of Biological Sciences, Indian Institute of Science Education and Research (IISER) Mohali, Punjab \\ 140306, India, ${ }^{2}$ Centre for Neuroscience, Indian Institute of Science, Bangalore 560012, India, and ${ }^{3} \mathrm{National}$ Brain \\ Research Centre, Manesar, Nainwal Mode, Gurgaon 122051, India
}

\begin{abstract}
The G-protein (heterotrimeric guanine nucleotide-binding protein)-coupled receptors (GPCRs) in the olfactory system function to sense the surrounding environment and respond to various odorants. The genes coding for olfactory receptors in Caenorhabditis elegans are larger in number in comparison to those in mammals, suggesting complexity in the receptor-odorant relationships. Recent studies have shown that the same odorant in different concentrations could act on multiple receptors in different neurons to induce attractive or repulsive responses. The ASH neurons are known to be responsible for responding to high concentrations of volatile odorants. Here, we characterize a new GPCR, SRX-97. We found that the srx-97 promoter drives expression specifically in the head ASH and tail PHB chemosensory neurons of C. elegans. Moreover, the SRX-97 protein localizes to the ciliary ends of the ASH neurons. Analysis of clustered regularly interspaced short palindromic repeats (CRISPR)-based deletion mutants of the srx-97 locus suggests that this gene is involved in recognition of high concentrations of benzaldehyde. This was further confirmed through rescue and neuronal ablation experiments. Our work brings novel insights into concentration-dependent receptor function in the olfactory system, and provides details of an additional molecule that helps the animal navigate its surroundings.
\end{abstract}

Key words: ASH neuron; benzaldehyde; C. elegans; SRX-97

\section{Significance Statement}

Although G-protein-coupled receptors (GPCRs) have been known to function as chemosensory receptors, the expression pattern and function of a large number of GPCRs remains unknown. This work sheds light on the expression pattern of an uncharacterized GPCR, SRX-97. Our work shows that this protein is expressed very specifically in two sensory neuron pairs in the head and tail region and is required for concentration dependent sensing of odors in Caenorhabditis elegans.

\section{Introduction}

Animals sense a wide range of volatile and water-soluble chemicals through their olfactory system. The olfactory system consists of several neurons that express different sets of seven-transmembrane G-protein-coupled receptors (GPCRs). The odorant binds to the GPCRs, activating distinct intracellular signaling pathways and thus directing the animal's

Received January 9, 2020; accepted December 18, 2020; First published January 4, 2021.

The authors declare no competing financial interests. response to different external cues (for review, see Katritch et al., 2013; Erlandson et al., 2018).

Caenorhabditis elegans are soil-dwelling animals that possess well-developed chemosensory systems for their survival. They perceive their environment through various sensory neurons to find food sources, mates, and to escape from dangerous conditions. In C. elegans, 13 pairs

Author contributions: N.Y.K., A.G.-R., and K.B. designed research; N.Y.K., S.B., and S.K. performed research; N.Y.K. and S.B. analyzed data; N.Y.K. and K.B. wrote the paper. 
of chemosensory neurons carry out the majority of chemosensation as they express around 1300 functional chemosensory GPCRs (csGPCRs; Robertson and Thomas, 2006; Vidal et al., 2018). This diversity of csGPCRs allows the animal to discriminate between different odors. Thus, the specific expression of any GPCR or combined expression of different GPCRs on a specific neuron or in multiple neurons can modulate the animal's perception toward the same odorant.

The olfactory neurons that are involved in sensing a large number of attractive cues are the AWA and AWC neurons. These two pairs of neurons are involved in chemotaxis to various chemicals like diacetyl (DA), isoamyl alcohol (IAA), pyrazine, benzaldehyde, and butanone (Bargmann et al., 1993; Troemel et al., 1995; Colbert et al., 1997). The avoidance behavior toward the repellents nonanone and 1-octanol is mediated through the sensory neurons AWB, ASH, and ADL (Troemel et al., 1997; Chao et al., 2004). Besides this, many volatile chemicals detected by olfactory neurons could act as attractants at low concentrations and repellents at high concentrations (Yoshida et al., 2012). For example, at low concentrations, DA is sensed by the GPCR ODR-10, in the AWA neuron acting as an attractant (Sengupta et al., 1996), whereas at high concentration it is sensed by the SRI-14 GPCR in the ASH neurons and acts as a repellent (Taniguchi et al., 2014). Additionally, ASH neurons are polymodal neurons involved in avoidance behaviors toward different nociceptive signals, like noxious chemicals, nose touch, hyperosmolarity, and volatile repellents (Hilliard et al., 2005; Bargmann, 2006). The ASH neurons convey information through multiple receptors. For example, nose touch has been shown to be detected by transient receptor potential (TRP) channel proteins like OSM-9 and OCR-2 (Colbert et al., 1997; Tobin et al.,

This work was supported by Department of Biotechnology (DBT)/Wellcome Trust India Alliance Fellowships Gants IAVS/19/2/504649 and IAVI/12/1/500516 (to K.B.) and partially supported by DBT, Ministry of Human Resource DevelopmentScheme for Transformational and Advanced Research in Sciences and Department of Science and Technology-Science and Engineering Research Board Grants BT/PR24038/BRB/10/1693/2018, STARS/APR2019/BS/454/FS, and SERB/F/7047 as well as a DBT-IISc Partnership Grant (K.B.). Anindya GhoshRoy lab is supported by the National Brain Research Centre core fund from the Department of Biotechnology and the DBT/Wellcome Trust India Alliance Fellowship Grant IA/I/13/1/500874 (to A.G.-R.). A number of strains were provided by CGC, which is funded by the National Institutes of Health Office of Research Infrastructure Programs Grant P40 OD010440. N.Y.K. was funded by the Council of Scientific and Industrial Research (CSIR)-University Grants Commission (UGC) for a Graduate Fellowship. S.B. was funded by a Kisehore Vaigyanik Protsahan Yojanana Fellowship for undergraduate students.

Acknowledgements: We thank Yogesh Dahiya for help with making the srx97 deletion strain. We also thank Ankit Negi for routine work, Pratima Pandey and Umer Saleem Bhat for help with cloning, and the IISER Mohali Confocal facility for use of the confocal microscope. A.G.-R. thanks Harjot Kaur for help with two-photon microscopy.

Correspondence should be addressed to Kavita Babu at kavita.babu@ babulab.org/kavitababu@iisc.ac.in or Nagesh Kadam at nagesh.kadam@ babulab.org.

https://doi.org/10.1523/ENEURO.0011-20.2020 Copyright (c) 2021 Kadam et al.

This is an open-access article distributed under the terms of the Creative Commons Attribution 4.0 International license, which permits unrestricted use, distribution and reproduction in any medium provided that the original work is properly attributed.
2002), while hyperosmolarity is detected by OSM-10 (Hart et al., 1999). The ASH neurons forms strong synaptic connections with the AVA command interneurons, which regulates the backward locomotion of $C$. elegans (Gray et al., 2005; Zheng et al., 2012; Pokala et al., 2014; Bhardwaj et al., 2018, 2020). Thus, activation of ASH neurons can affect backward locomotion or avoidance behaviors in C. elegans.

ASH neurons are also reported to be involved in sensing undiluted or high concentrations of benzaldehyde (Troemel et al., 1995; Walker et al., 2009; Aoki et al., 2011; Taniguchi et al., 2014). Here, we show that SRX-97 is expressed in the ASH neurons. We have used the clustered regularly interspaced short palindromic repeats (CRISPR)/Cas9 method for genome editing and have made a deletion in the srx-97 gene locus, generating a null mutation in srx-97. The srx-97 mutants present defects in chemotaxis behavior, more specifically toward high concentrations of benzaldehyde. Moreover, the mutant phenotype could be rescued by both endogenous and neuron-specific expression of the wild-type (WT) srx-97 gene, suggesting concentration-dependent behavioral plasticity for odors in $C$. elegans through the SRX-97 GPCR.

\section{Materials and Methods}

\section{C. elegans strains and maintenance}

All C. elegans strains were maintained on nematode agar growth media (NGM) plates seeded with OP50 Escherichia coli at $20^{\circ} \mathrm{C}$ under standard conditions (Brenner, 1974). The C. elegans, N2 (Bristol strain) was used as the WT control, and the mutant strains CX2205 odr-3 (n2150) V, CX10 osm-9 (ky10) IV, NL792 gpc-1(pk298) X, RB2464 tax-2 (ok3403) I and VC3113 tax-4 (ok3771) III, as well as the AWC ablated strain PY7502 oyls85 (Pceh-36::TU\#813 + Pceh-36:: TU\#814 + Psrtx-1::GFP + Punc-122::DsRed, TU\#813 and TU\#814 are split caspase vectors) used in this study were obtained from the Caenorhabditis Genetic Centre (CGC). Double mutants were made through standard genetic procedures and verified using PCR. The list of primers used for PCR verification in this study is tabulated in Table 1. The strains used in this study are listed in Table 2.

\section{Rescue constructs and transgenes}

All constructs for the rescue of the srx-97 phenotype were generated using standard cloning methods (Sambrook and Russell, 2001). The pPD49.26 and $p P D 95.75$ vectors were used to clone the constructs. The primers used for cloning are indicated in Table 1. The transgenic strains were generated using standard microinjection techniques as described previously (Mello et al., 1991; Mello and Fire, 1995). The pCFJ90 and pPD95.75 plasmids were used to amplify or clone mCherry and GFP, respectively. The rescue constructs or promoter fusion constructs were injected at

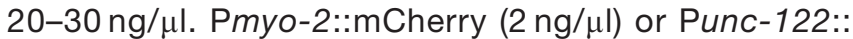
GFP $(25 \mathrm{ng} / \mu \mathrm{l})$ were used as co-injection markers. The constructs used in this study are described in Table 3. 
Table 1: Primers used in this study

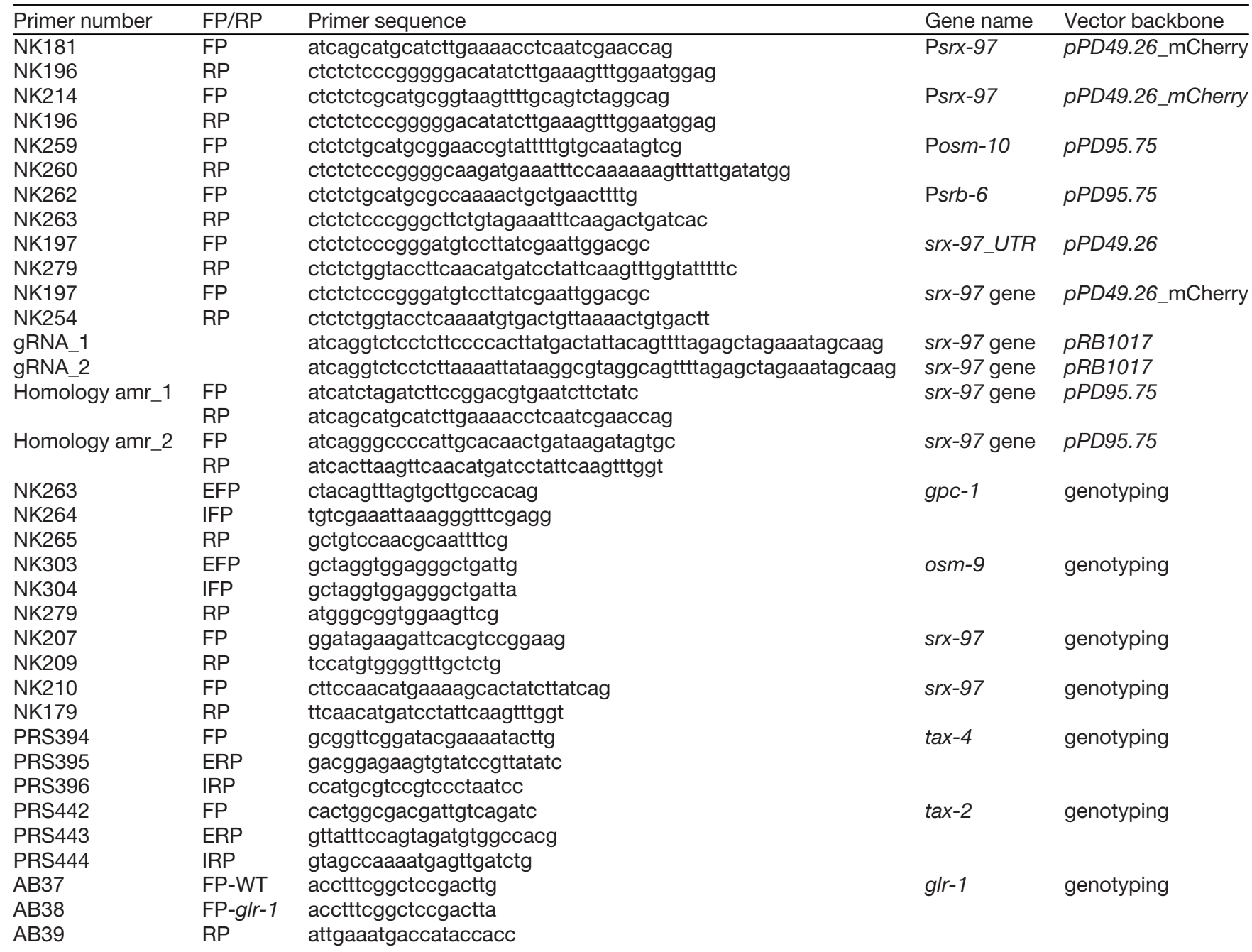

FP, forward primer; RP, reverse primer; FP-WT, external forward primer for WT glr-1 sequence; FP-glr-1, external forward primer for glr-1 sequence in the glr-1 point mutation line.

\section{Imaging experiments}

Young adult animals were used for imaging. The animals were immobilized with $30 \mathrm{mg} / \mathrm{ml} \mathrm{2,} \mathrm{3-butanedione}$ monoxamine (BDM) on $2 \%$ agarose pads in M9 media. The promoter mCherry images for $\mathrm{ASH}$ and $\mathrm{PHB}$ were acquired on a Leica SP6 upright laser scanning confocal microscope using the $40 \times$ oil-immersion objective lens. Laser lines from He-Ne (594) with HyD detectors were used to image fluorescence in the head and tail regions. All other imaging experiments were performed with oil immersion $40 \times / 1.4,63 \times / 1.4$ or $100 \times / 1.4$ plan Apochromat objectives using a Zeiss AxioCam MRm CCD camera on the Zeiss Axiolmager Z2 microscope.

\section{Behavioral assays \\ Chemotaxis assay}

The chemotaxis assay was performed using young adult $C$. elegans. Young adult animals were obtained by bleaching gravid adults and incubating the remaining eggs for $72 \mathrm{~h}(\mathrm{~h})$ at $20^{\circ} \mathrm{C}$. All chemotaxis assays were performed with standard $90 \mathrm{~mm}$ petriplates containing $15-18 \mathrm{ml}$ of chemotaxis medium (Agar, $1 \mathrm{M} \mathrm{MgSO}_{4}, 1 \mathrm{M}$ $\mathrm{CaCl}_{2}$, and $1 \mathrm{M} \mathrm{KPO}_{4} ; \mathrm{pH}$ 6.6). Wherever required, odorants were diluted in ethanol and reported as a percent by volume. Modified 90-mm quadrant plate chemotaxis assays were performed as described previously (Bargmann et al., 1993; Margie et al., 2013). Briefly, 5 min before the assay, $1 \mu \mathrm{l}$ of $0.5 \mathrm{~m}$ sodium azide was applied on four spots that were each $3 \mathrm{~cm}$ from the loading center. Sodium azide acts as an anesthetic agent to immobilize animals that reach the vicinity of the spot during the assay. A total of 50-150 animals were placed at the center of the plate between the four spots, $2 \mu$ l of ethanol were placed at the two-control spots and $2 \mu$ l of the test odorant were placed at the two-test spots. After 90 min of chemotaxis, animals within each sector were counted, and the chemotaxis index (C.I.) was calculated as the number of animals in the two test sectors minus the number of animals in the two control sectors, divided by the total number of animals on the plate excluding those that were not moving at the center of the 
Table 2: List of strain used in this study

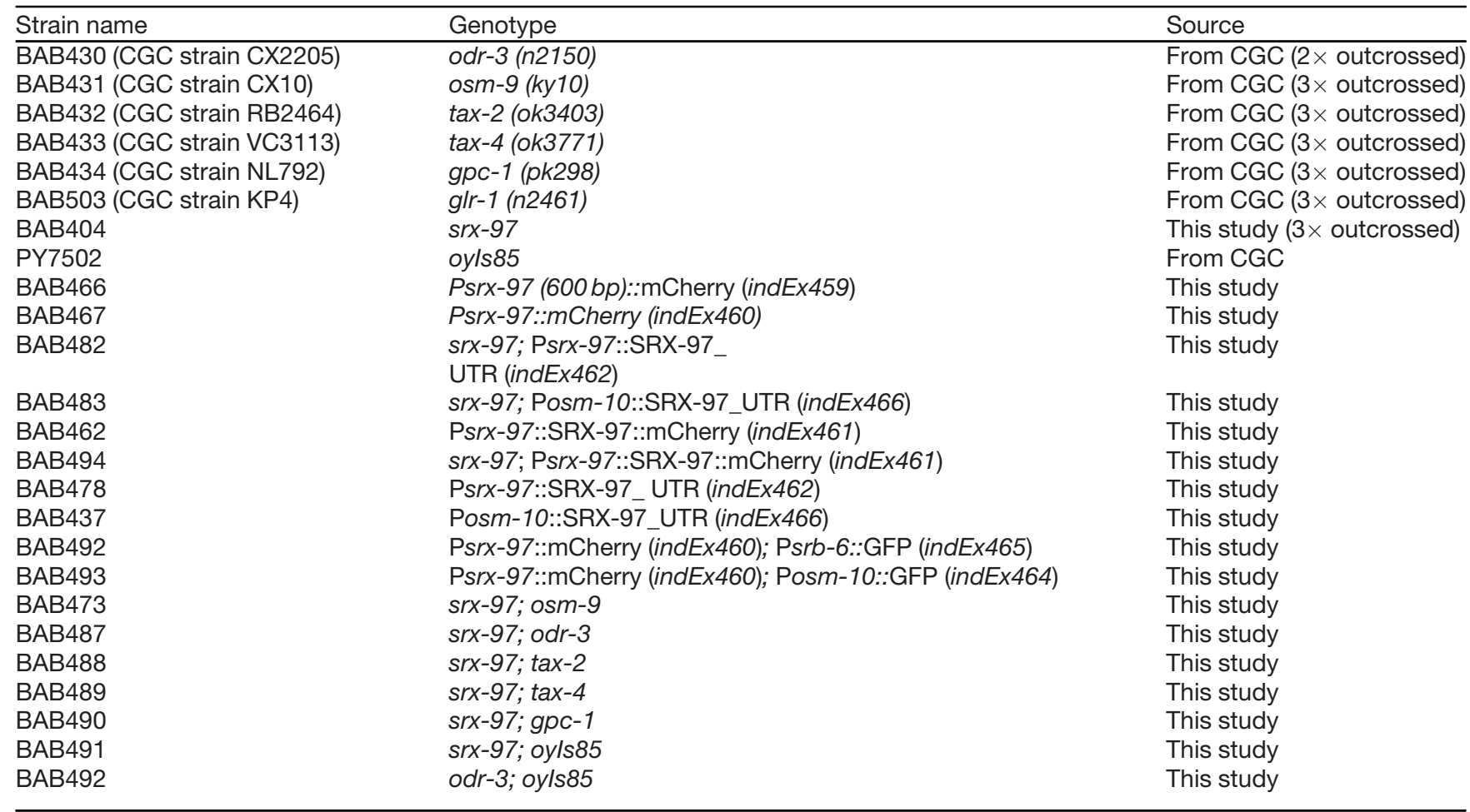

plate (illustrated in Extended Data Fig. 3-1A). A positive C.I. indicates an attraction to the chemical, and a negative C.I. indicates a repulsion to the chemical.

\section{Assay to evaluate chemotaxis frequency}

For analysis of the frequency or number of animals chemotaxing toward the source of benzaldehyde, a modified grid chemotaxis plate was used (Nuttley et al., 2001). The sodium azide was omitted so that animals could leave a spot after an initial approach. This grid consisted of four parallel lines drawn $1 \mathrm{~cm}$ apart to divide the plate area into five sectors, with the distance between the second and third lines being $2 \mathrm{~cm}$ (illustrated in Fig. 4A). Two microliters of benzaldehyde were placed on one small sheet of Parafilm, and the same amount of ethanol was placed on another as a control. The benzaldehyde and ethanol were placed at opposite ends of the plate (6 cm away). After a 60-min time interval, animals were immobilized by cooling the plates for $3 \mathrm{~min}$ at $-30^{\circ} \mathrm{C}$, and the plates were maintained at $4^{\circ} \mathrm{C}$ until counting. The number of animals in sectors a-d, with the test odorant being in a and $\mathrm{d}$, were counted and the kinetic C.I. was calculated as (number of animals in a + number of animals in b) - (number of animals in c + number of animals in $\mathrm{d}$ )/(total number of animals on the plate), yielding a C.I. range between +1.0 and -1.0 (illustrated in Fig. 4A). The animals that had crawled up the sides of the plate were excluded from the analysis. The score of 50-150 animals for each plate was used as one data point.

\section{Dry drop avoidance assay}

A drop of a solution containing the test chemicals [SDS, quinine, $\mathrm{CuSO}_{4}$, glycerol, and dihydrocaffeic acid (DHCA)] dissolved in M13 buffer (30 mm Tris, $100 \mathrm{~mm} \mathrm{NaCl}$, and 10 $\mathrm{mm} \mathrm{KCl}$ ) was delivered on the agar plate (NGM unseeded) 0.5-1 mm anterior to the moving animals (Hilliard et al., 2002). Once the animal encountered the dry drop of chemical, the head amphid neurons sensed the chemical triggered repulsion/avoidance behavior. The delayed response in seconds from the initial contact to a reversal was calculated in the assay. Videos were recorded for $1 \mathrm{~min}$ at $10 \mathrm{frames} / \mathrm{s}$ with one to two readings leaving a gap of 20-30 s between each trial. The graphs were plotted by taking the average value from two trials with $>30$ animals being analyzed for each condition over multiple days. If the animal failed to respond within $6 \mathrm{~s}$, the reversal time was considered as $6 \mathrm{~s}$. Drops of M13 buffer were used as a control where animals as expected did not show robust responses. Glass capillaries $(10 \mathrm{~mm})$ pulled by hand on flames to reduce the diameter of the tip were used to deliver the drops. The results were plotted using GraphPad Prism

Table 3: Plasmids used in this study

\begin{tabular}{lll}
\hline S. no. & Plasmid ID & Plasmid \\
\hline 1 & pBAB459 & Psrx-97(600 bp)::mCherry \\
2 & pBAB460 & Psrx-97::mCherry \\
3 & pBAB461 & Psrx-97::SRX-97::mCherry \\
4 & pBAB462 & Psrx-97::SRX-97_UTR \\
5 & pBAB464 & Posm-10::GFP \\
6 & pBAB465 & Psrb-6::GFP \\
7 & pBAB466 & Posm-10::SRX-97_UTR \\
8 & pBAB472 & srx-97_homology arms \\
9 & pBAB470 & gRNA_1 \\
10 & pBAB471 & gRNA_2 \\
\hline
\end{tabular}


V6 and evaluated using one-way ANOVA. The mean \pm SEM were plotted.

\section{Nose touch assay}

The response to nose touch was analyzed on unseeded plates as described previously (Kaplan and Horvitz, 1993). Briefly, young adult animals were placed on NGM plates and allowed to habituate for $1 \mathrm{~min}$. An eyelash was placed in the path of the forward moving animal, and those who showed a reversal of the body movement on collision with the eyelash were considered as positive responders. The experiment was performed with 20-30 animals per genotype over multiple days. The analysis was performed using 10 trials/animal, and the data are shown as the percentage of positive responders.

\section{Aldicarb assay}

The aldicarb assays were performed as described previously (Mahoney et al., 2006). Briefly, Aldicarb plates were made the previous day by adding $100 \mathrm{~mm}$ stock solution (prepared in ethanol) of aldicarb (Sigma-Aldrich) to molten NGM at a final concentration of $1 \mathrm{~mm}$. Plates were then seeded with OP50 E. coli and stored in dark at room temperature overnight. For each assay $20-25$ young adult animals were transferred on to the aldicarb plates and scored for paralysis every $10 \mathrm{~min}$ for up to $120 \mathrm{~min}$. Animals were considered paralyzed when they failed to show body bends following prodding three times on the head.

\section{Ablation of ASH neurons}

ASH neuronal ablation experiments were performed to test the benzaldehyde chemotaxis dependence on this neuron, which was tagged with Psrx-97::mCherry. L2 staged animals were used for the ablation experiment, as ablations are more effective in early stages (Avery and Horvitz, 1987, 1989; Bargmann and Horvitz, 1991). During ablation and imaging, the animals were immobilized on $5 \%$ agarose pads with $0.1-\mu \mathrm{m}$-diameter polystyrene beads (00876-15; Polystyrene suspension). The Bruker Corporations ULTIMA setup was used to perform twophoton imaging and ablations simultaneously (Basu et al., 2017). A 60× water immersion objective was used for ablation and imaging experiments, GFP and mCherry were visualized using 920- and 1040-nm lasers. A shot for 60 ms pulsed femtosecond IR laser [pulse width $80 \mathrm{fs,} \mathrm{ir-}$ radiation pulse width: $50 \mathrm{~ms}$, laser point spread function (PSF) $400 \mathrm{~nm}$ and $z$-axis PSF-1.5um and wavelength of the laser $720 \mathrm{~nm}$ ] was used for all ablation experiments. Animals were then examined for successful ablation under a fluorescence microscope. These animals were allowed to grow and recover until they reached the young adult stage. Single animals were then transferred to individual unseeded plates and allowed to habituate for $1 \mathrm{~min}$. Benzaldehyde (concentration of $10^{-1}$ ) was filled in the glass capillary having a small opening pore. The filled capillary was held just in front of the anterior region of the forward moving animals. Videos were recorded for $5 \mathrm{~min}$ at $10 \mathrm{frames} / \mathrm{s}$ with five to six trials leaving a gap of $\sim 1$ min between each reading. Graphs were plotted by taking the average value from five to dix trials, with around
25 animals analyzed for each condition over multiple days. The results were plotted using GraphPad Prism V6 and evaluated using one-way ANOVA. The mean \pm SEM was plotted.

\section{CRISPR/Cas9 mediated deletion of the srx-97 gene}

The CRISPR/Cas9 system was used to create the srx97 deletion mutation, as described previously (Dickinson and Goldstein, 2016). The two guide RNAs were designed (Hsu et al., 2013) and cloned separately into the pRB1017 vector under the CeU6 promoter. The Cas 9 enzyme was expressed from the pJW1259 vector under the erf-3 promoter. The selection excision cassette (SEC) containing plasmid $p D D 287$ was cloned along-with flanking loxP sites into the pPD95.75 vector as described previously (Dahiya et al., 2019). The resulting plasmid was used to clone homology arms (500-600 bp) using restriction enzyme-based cloning methods.

The plasmid mixture containing repair template $(40 \mathrm{ng} / \mu \mathrm{l})$, sgRNA_1 (10 ng/ $\mu \mathrm{l})$, sgRNA_2 (10 ng/ $/ \mathrm{ll}), p J W 1259(50 \mathrm{ng} / \mu \mathrm{l})$, pCFJ90 $(2.5 \mathrm{ng} / \mu \mathrm{l})$, and Pvha-6::mCherry $(15 \mathrm{ng} / \mu \mathrm{l})$ was injected into 20-30 adult hermaphrodite animals (containing four to five eggs) that were kept at $20^{\circ} \mathrm{C}$. Hygromycin was added after $60 \mathrm{~h}$ of injection, directly on the NGM plate containing $C$. elegans. The hygromycin treated plates were left for $10 \mathrm{~d}$ at $20^{\circ} \mathrm{C}$. Next, $20-30$ non-fluorescent rollers were singled out on regular seeded NGM plates. Once 100\% roller progeny were observed on the plates, these plates were kept at $34^{\circ} \mathrm{C}$ for $3-4 \mathrm{~h}$. Normal moving $\mathrm{C}$. elegans were then picked and allowed to produce progeny. The genomic DNA was isolated from these progenies and the desired deletion was confirmed using PCR and sequencing techniques.

\section{Statistical analysis}

All statistical analyses on behavioral assays were performed by using GraphPad Prism version 6.0. The error bars represent SEM. Statistical significance was determined using one-way ANOVA along with the Sidak's post hoc test for multiple comparisons. Asterisks in the graphs indicate that the mean differences were statistically significant. The levels of significance were set as ${ }^{*} p<0.05$, ${ }^{* \star} p<0.01,{ }^{* * *} p<0.001$

\section{Results}

\section{The Psrx-97::mCherry transgene presents unique expression in the ASH and PHB chemosensory neurons}

csGPCRs are categorized into nine different classes based on their sequence homology with the Rhodopsin class of molecules (Fredriksson et al., 2003; Lagerstrom and Schioth, 2008). The C. elegans genome has 1341 genes coding for GPCRs; however, the expression pattern of only 320 genes is known at a single-cell resolution (Robertson and Thomas, 2006; Taniguchi et al., 2014; Vidal et al., 2018). Reports suggest that GPCRs are also expressed in non-neuronal tissues like intestine and are involved in sensing internal cues (Vidal et al., 2018). Some GPCRs change their expression pattern once the animal encounters starvation or dauer-ike conditions (Vidal et al., 
2018). Despite a large number of studies on the functional and spatial diversity of GPCRs, the expression pattern and function for a majority of the csGPCRs are still unknown (Robertson and Thomas, 2006; Taniguchi et al., 2014; Vidal et al., 2018).

We started this study to gain insight into the function of a previously uncharacterized GPCR, SRX-97. SRX-97 was identified in an aldicarb-based RNAi screen and was found to be hypersensitive to aldicarb (Babu et al., 2011; Babu et al., unpublished data). To determine the expression pattern of SRX-97, a region $2 \mathrm{~kb}$ upstream of the predicted translational start codon of the srx-97 gene along with six base pairs of the first exonic region were used as a promoter to generate the Psrx-97::mCherry reporter line. In these transgenic animals, mCherry expression was specifically detected in a single pair of head amphid neurons and a single pair of tail neurons (Fig. $1 A, B, D, E$ ). Moreover, even a 600-bp region upstream of the $s r x-97$ gene with six base pairs from the first exon showed a similar expression pattern as the 2-kb promoter (Fig. 1C). No expression was detected in any other part of the body. Since the amphid and phasmid neurons are involved in chemotaxis, our data suggested that SRX-97 could specifically be involved in these neurons and likely in chemosensory signaling.

Next, we began identifying Psrx-97:: mCherry expressing neurons based on their cilium morphology, the cell body position in the head and tail region, and colocalization experiments. To uncover the neurons that showed expression of the srx-97 promoter, we generated the Psrb-6::GFP transgenic line, which shows expression in the ASH and ADL neurons in the amphid region (Troemel et al., 1995). The Psrx-97:: mCherry line presented colocalization in a single neuron pair with the Psrb-6::GFP line (Fig. $1 F$, top panel), indicating that Psrx-97::mCherry could be expressed in either the ASH or the ADL neurons. To conclusively identify the Psrx-97:: mCherry expressing neuron, we generated another transgenic line with Posm-10::GFP, which shows expression in the amphid ASH and ASI neurons and the PHAPHB neurons in the phasmid region (Fig. 1F, bottom panel) and (Hart et al., 1999). The colocalization of Psrx-97::mCherry in a single amphid neuron pair with both marker lines indicated that the $s r x-$ 97 promoter drives expression in the ASH neurons. In line with a recent report that suggests that $50 \%$ of GPCRs which express in ASH neurons also show expression in the PHB neuron (Vidal et al., 2018), we found that in the tail region, Psrx-97::mCherry showed colocalization with a pair of phasmid neurons (Fig. 1G, top panels indicating colocalization with one phasmid neuron). Based on the orientation of the animal (posterior right and ventral down), this neuron appears to be the PHB neuron (Fig. 1G, DIC image in bottom panel).

We then analyzed a SRX-97 translational reporter and found that the Psrx-97::SRX-97::mCherry transgenic line showed SRX-97 protein localization toward the cilium tip of the ASH neurons (Fig. $1 H$ ), indicating that this protein may be involved in sensing environmental cues from the surroundings.

\section{CRISPR/Cas9 mediated deletion of srx-97}

C. elegans have 13 pairs of chemosensory neurons in the anterior amphid and posterior phasmid regions. However, it can detect several different chemical cues ranging from volatile to water-soluble odorants through diverse GPCRs (Robertson and Thomas, 2006; Vidal et al., 2018). Seven percent of the $C$. elegans genome encodes for chemoreceptors. However, only around 900 chemoreceptor genes have been characterized functionally, many through RNAi experiments (Robertson and Thomas, 2006; Taniguchi et al., 2014; Vidal et al., 2018). Hence, only a few mutant lines of GPCRs are available. Our studies have shown that the srx-97 promoter drives expression in ASH and PHB neurons. This expression pattern raised the possibility that it might function as a receptor for odorant/s. Since no mutant strain was available for this gene, we used the CRISPR/Cas9 based strategy to generate a deletion in the srx-97 gene.

The SRX-97 GPCR is part of the SRX family of proteins that belong to the SRG superfamily that encodes around 320 genes (Robertson and Thomas, 2006; Vidal et al., 2018). The srx-97 gene encodes a predicted protein of 317 amino acids (Fig. 2A). Hydrophobicity analyses showed that the SRX-97 protein encodes for a seventransmembrane domain protein, with the characteristic topology of GPCRs (Fig. 2A). By using the gene-editing CRISPR/Cas9 technique, we made a complete deletion of the srx-97 gene (from $61 \mathrm{bp}$ of the first exon to the $3^{\prime}$ UTR region, deleting a 1834-bp sequence; Fig. $2 B, C$ ). We next performed aldicarb assays with the $s r x-97$ deletion animals and found no significant difference in aldicarb sensitivity between the deletion strain and WT control animals (Extended Data Fig. 2-1A). These data, along with the localization and expression pattern of SRX-97, indicated that SRX-97 likely did not function at the neuromuscular junction to cause defects in aldicarb sensitivity. The aldicarb phenotype in the RNAi screen could have been a false positive because of cross-complementation reactions with other GPCRs. Hence, we proceeded to study the role of SRX-97 in other processes.

\section{Loss of srx-97 leads to defects in chemotaxis toward benzaldehyde}

ASH is a polymodal neuron that can respond to noxious, mechanical and osmotic stimuli (Kaplan and Horvitz, 1993; Colbert et al., 1997; Hilliard et al., 2004, 2005). To characterize the role of the SRX-97 GPCR in ASH neurons, we examined the response of the srx-97 mutant line toward several compounds including glycerol, SDS, $\mathrm{Cu}^{2+}$, quinine, DHCA, and acetic acid (Kaplan and Horvitz, 1993; Hilliard et al., 2002, 2005). The srx-97 mutant animals showed minor defects in avoidance behavior toward $2 \mathrm{~m}$ glycerol and $100 \mathrm{~mm}$ DHCA when compared with the control WT animals (Fig. $3 A$; Table 4). We also tested the behavior of $s r x-97$ mutants to other chemicals and found that $s r x-97$ did not show significant differences when compared with WT control animals in their behavior toward glycerol $(1 \mathrm{M})$, SDS $(0.1 \%$ and $1 \%), \mathrm{Cu}^{2+}(1 \mathrm{~mm}$ and $10 \mathrm{~mm})$, quinine (1 $\mathrm{mm}$ and $10 \mathrm{~mm}), \mathrm{DHCA}(1 \mathrm{~m})$, and acetic acid ( 1 and $0.1 \mathrm{~m}$; Fig. 3B,C; Table 4). Mutants in odr-3, a $\mathrm{G} \alpha$ protein, were used as controls for these avoidance assays, since odr-3 has been reported to be involved in multiple behaviors controlled by the ASH neurons (Roayaie et al., 1998; Hilliard et al., 2004, 2005; 
A

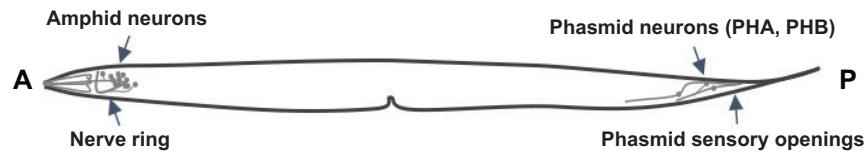

B

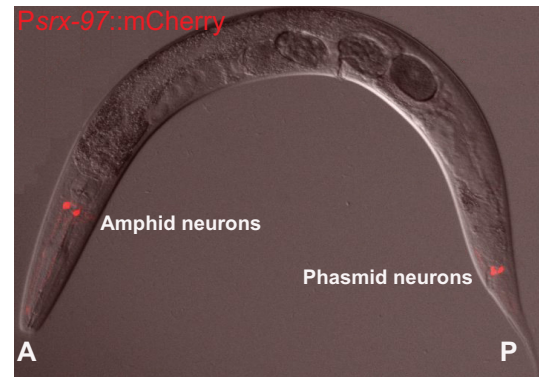

D

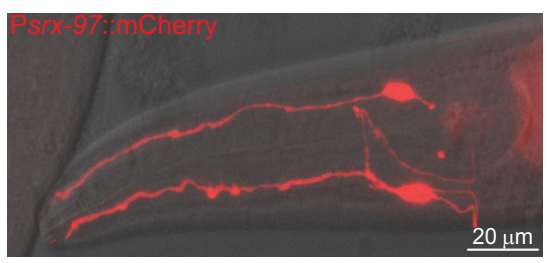

C

E
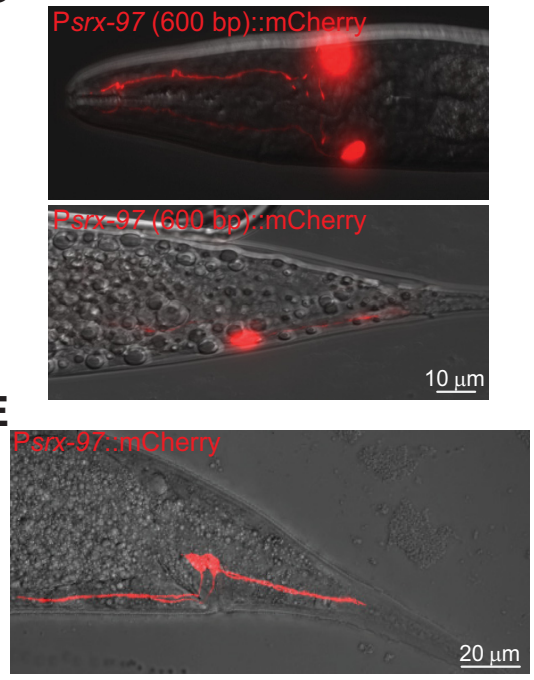

$F$

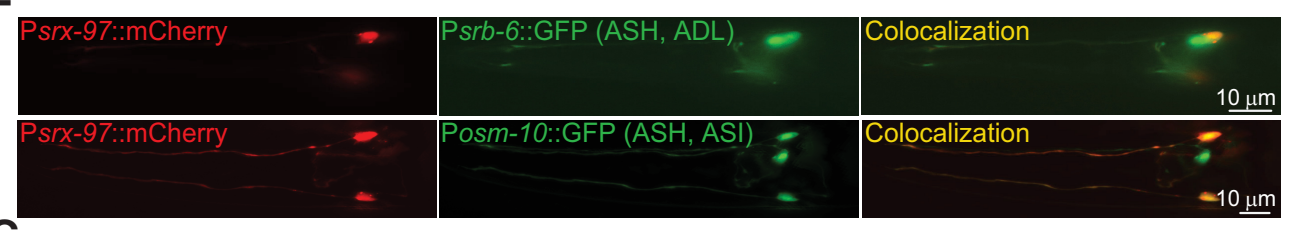

G

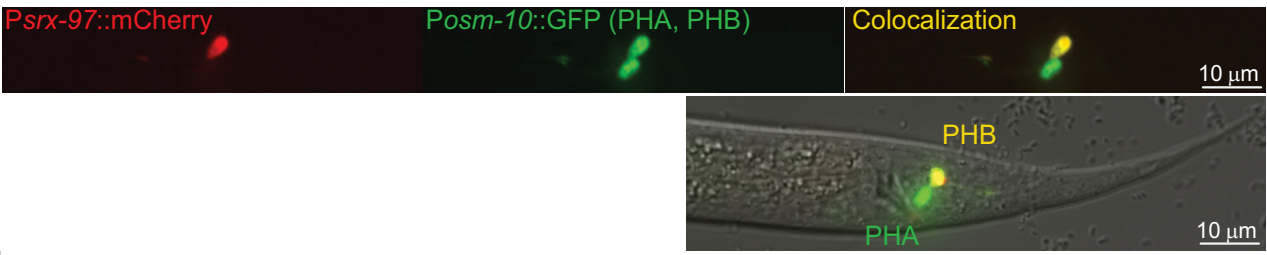

H
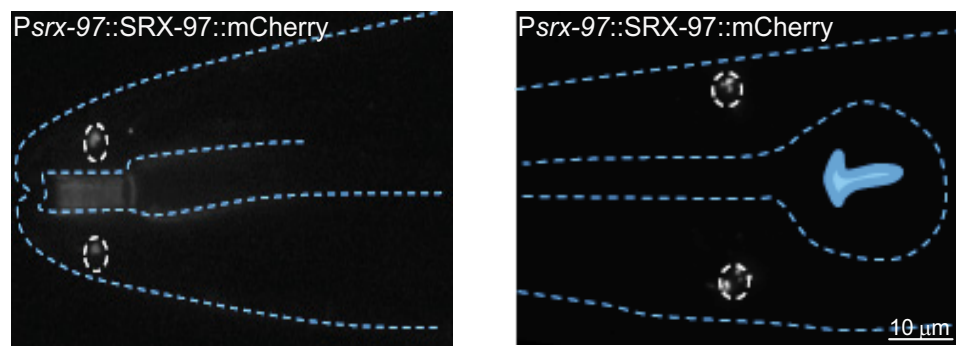

Figure 1. Expression of Psrx-97::mCherry in ASH and PHB neuron. $\boldsymbol{A}$, Cartoon image showing the location of the amphid and phasmid neurons in C. elegans. B, Expression of the Psrx-97::mCherry transgenic construct in the whole animal. $\boldsymbol{C}$, srx- 97 promoter (600 bp) expression in a single pair of amphid and phasmid neurons. $\boldsymbol{D}$, The expression of the srx-97 promoter (2 kb) in a pair of amphid neurons and $(\boldsymbol{E})$ phasmid neurons in $C$. elegans. $\boldsymbol{F}$, Expression of Psrb-6::GFP and Posm-10::GFP in their respective neurons (indicated on the figure) and their co-localization with Psrx-97::mCherry in the amphid ASH neurons. G, Expression of Posm-10:: GFP in its respective neurons (indicated on the figure) and their co-localization with Psrx-97::mCherry in the phasmid PHB neuron. The lower panel indicates a DIC image indicating the position of the PHA and PHB neurons. $\boldsymbol{H}$, Expression of SRX-97::mCherry in the cell bodies (dotted circles) and SRX-97 localization to the cilium tip of the ASH neurons (between the dotted circles in the figure to the left). 
A

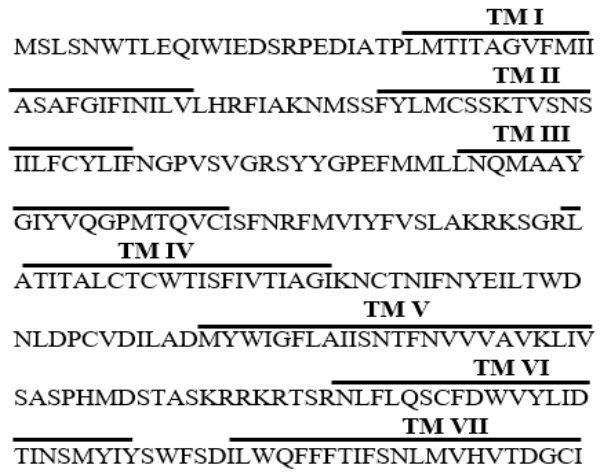

MLFFNYEGKKKTLTLTLNNVKREVTVLTVTF

B

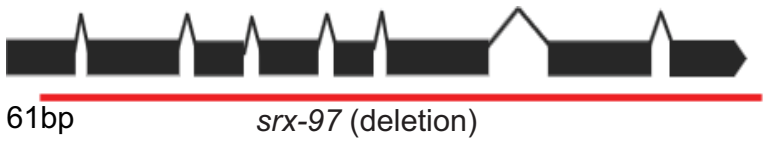

C

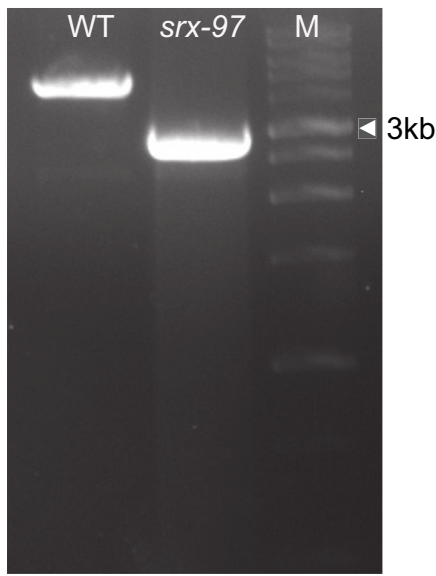

Figure 2. The SRX-97 transmembrane domain and CRISPR/ Cas9 generated mutation of $s r x-97$. A, Amino acid sequence showing the predicted seven transmembrane domain of SRX97. $\boldsymbol{B}$, Exonic structure of the srx-97 gene with the red line showing the CRISPR/Cas9 deletion obtained. The deletion encompasses the gene from the 61st base pair to the 1895th base pair including part of the $3^{\prime}$ UTR of the gene. $\boldsymbol{C}$, Amplification of the chromosomal region showing the deletion of the srx-97 gene (2730 bp) using CRISPR/Cas9 compared with control WT (4566 bp) gene. A 1-kb DNA ladder was used in the line marked Marker (M). Extended Data Figure 2-1 supports this figure.

Zhang et al., 2016). The ASH neurons are also known to play a role in sensing mechanical stimuli (Kaplan and Horvitz, 1993). In order to test the role of SRX-97 in mechanosensation, we performed a nose touch assay with WT, srx-97, and glr-1 (defective for nose touch assay; Maricq et al., 1995) animals. Upon performing the assays, we observed that srx-97 mutant animals did not show significant defects in nose touch assays when compared with WT controls (Fig. 3D). These data indicate that loss of srx-97 does not affect many aspects of the general ASH neuronal responses.
The ASH neurons are also known to be involved in detecting volatile chemicals (Troemel et al., 1995). To analyze the role of SRX-97 in detecting volatile chemicals, we used a modified chemotaxis plate, having four quadrants, two opposite quadrants for test solutions (T), and two for control solutions (C; illustrated in Extended Data Fig. $3-1 A$ ). Both control and test spots were $3 \mathrm{~cm}$ away from the C. elegans loading center. Before the addition of control or test solution, we added sodium azide to paralyze the animals once they reach their respective spots. Next, we calculated the C.I. by measuring the number of animals in each quadrant with the formula shown in Extended Data Figure 3-1A. Previous work has shown that in chemotaxis assays, the ASH neurons are involved in aversive behaviors toward the repellent 1-octanol (Chao et al., 2004). Here again, we found no significant change in the C.I. of the srx-97 mutant line when compared with WT control animals (Fig. $3 E$ ). Recent findings suggest that the ASH neurons are involved in sensing high concentrations of chemicals, such as IAA (Yoshida et al., 2012) and DA (Taniguchi et al., 2014). In the chemotaxis assays, we used a range of concentrations of IAA and DA, testing for any defects in responses toward these chemicals. We found that the srx97 mutants did not show any significant defects in chemotaxis toward IAA and DA when compared with control animals (Fig. 3F; Table 5).

The ASH neurons are also known to be involved in detecting benzaldehyde (Troemel et al., 1995; Walker et al., 2009; Aoki et al., 2011; Taniguchi et al., 2014). A previously identified GPCR, DCAR-1, has homology with the SRX family of proteins and dcar-1 mutants show defective chemotaxis toward undiluted benzaldehyde (Aoki et al., 2011). In our chemotaxis assays we found that the srx-97 mutant animals showed significantly more attraction to a high concentration of benzaldehyde $\left(10^{-1}\right)$ when compared with WT controls animals (Fig. $3 G$ ). We also found that as reported previously odr-3 mutant animals showed reduced attraction toward benzaldehyde (Fig. 3G; Roayaie et al., 1998). At low concentrations $\left(10^{-2}\right.$ and $10^{-3}$ ) of benzaldehyde and undiluted benzaldehyde, there was no significant difference between srx-97 and WT animals (Fig. 3G). Earlier reports indicate that the ASH neurons are involved in responding to high concentrations of benzaldehyde $(0.1 \% \mathrm{v} / \mathrm{v})$, whereas medium or low concentrations $(0.005-0.0001 \%)$ of benzaldehyde are sensed by the AWC and AWA neurons (Leinwand et al., 2015). Since SRX-97 is expressed in the ASH neurons, it could be involved in sensing a very high concentration range of benzaldehyde. In order to confirm the srx-97 mutant phenotype, we tried to rescue the defects seen in the srx-97 mutants. We found that the defects in chemotaxis toward benzaldehyde seen in the srx-97 animals could be rescued by expressing SRX-97 under its endogenous promoter, and partially rescued by expressing SRX-97 under the osm-10 promoter that drives expression in the $\mathrm{ASH}$ and ASI neurons (Fig. $3 H$ ). Although we observed rescue of the srx-97 mutants with the srx-97 and the osm-10 promoter lines, we also found a small but significant rescue in animals that did not carry any observable rescuing arrays, possibly because of low expression of the rescuing 
A

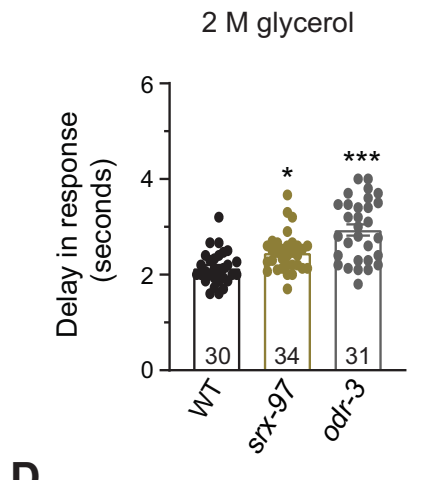

D

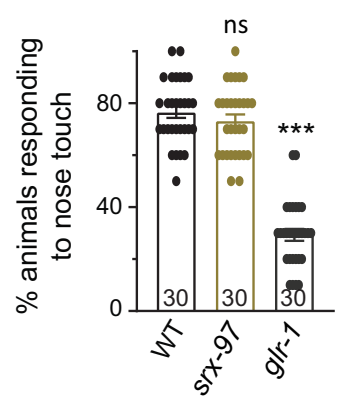

B

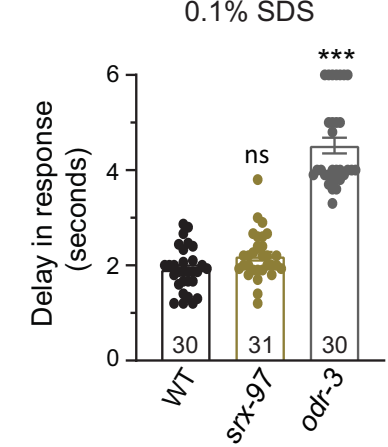

E

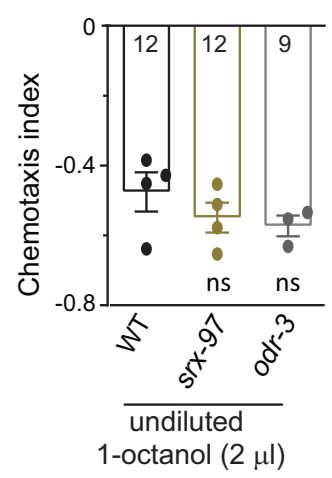

C

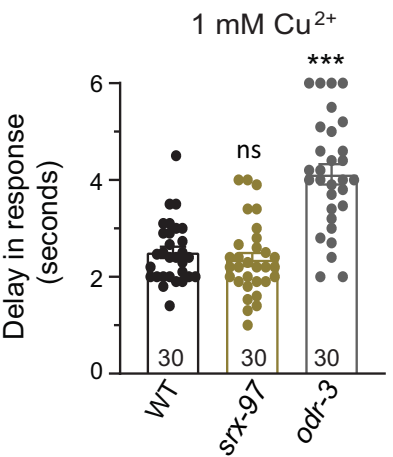

$\mathbf{F}$

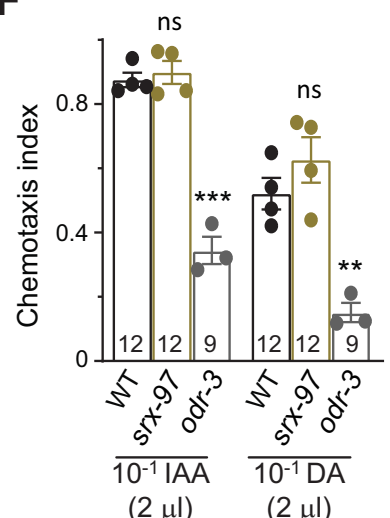

G

$\mathrm{H}$
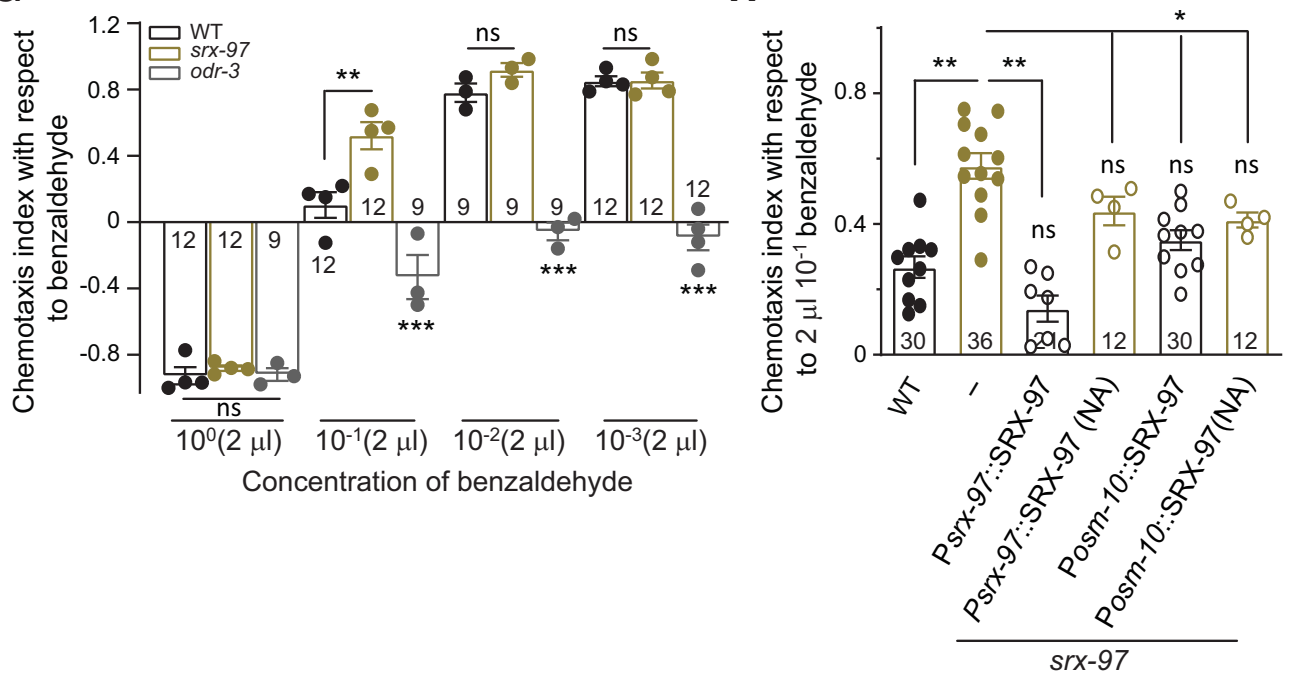

Figure 3. Behavior of $s r x-97$ mutant animals toward water-soluble and volatile chemicals. $\boldsymbol{A}$, Graph showing the delay in avoidance response toward a dry spot of $2 \mathrm{M}$ glycerol in WT, srx-97, and odr-3 mutant animals. The number of animals assayed for each genotype is indicated at the base of each plot for panels $\boldsymbol{A}-\boldsymbol{D}$. $\boldsymbol{B}$, Graph showing the delay in avoidance toward a dry spot of $0.1 \%$ SDS in WT, srx-97, and odr-3 mutant animals. C, Graph showing the delay in avoidance toward a dry spot of $10 \mathrm{~mm} \mathrm{CuSO}_{4}$ in WT, srx97, and odr-3 mutant animals. $\boldsymbol{D}$, Graph showing the percentage of avoidance on nose touch stimuli of WT, srx-97, and glr-1 mutant animals. The numbers at the base of graphs in $\boldsymbol{A}-\boldsymbol{D}$ indicate the number of animals tested for each genotype. $\boldsymbol{E}$, Graph indicating the negative chemotaxis indices of WT, srx-97, and odr-3 mutant animals toward the repellent octanol. The assay was done in triplicates over multiple days for all chmotaxis assays. Each dot indicates an assay done in triplicate for all graphs from $\boldsymbol{E}-\boldsymbol{H}$. $\boldsymbol{F}$, Chemotaxis indices toward high concentrations $\left(10^{-1}\right)$ of DA and IAA. $G$. Chemotaxis indices toward multiple concentrations of benzaldehyde. $\boldsymbol{H}$, Chemotaxis indices toward high concentrations of benzaldehyde in WT, srx-97, and rescue strains of srx-97. This rescue experiments used SRX-97 under its own promoter and under the osm-10 promoter. Animals that did not show expression of the arrays (NA, no array) were used as controls in these experiments. The error bars represent SEM, and statistical significance is represented as "ns" for not significant, ${ }^{*} p<0.05,{ }^{* *} p<0.01,{ }^{* * *} p<0.001$. The numbers at the base of each graph from $\boldsymbol{E}-\boldsymbol{H}$ indicates the total number of times the experiment was performed with 50-150 animals used in each trial. Extended Data Figure 3-1 supports this figure. 
Table 4: Response of srx-97 mutants toward multiple water-soluble chemicals

\begin{tabular}{|c|c|c|}
\hline Water-soluble chemicals & Genotype & Avoidance in seconds \\
\hline \multirow{3}{*}{$\begin{array}{c}\text { Glycerol } \\
(1 \mathrm{M})\end{array}$} & WT & $1.99 \pm 0.43(n=30)$ \\
\hline & $s r x-97$ & $2.42 \pm 0.17(n=33)$ \\
\hline & odr-3 & $3.53 \pm 0.25(n=30)^{\star \star \star}$ \\
\hline \multirow{3}{*}{$\begin{array}{l}\text { SDS } \\
(1 \%)\end{array}$} & WT & $1.72 \pm 0.23(n=30)$ \\
\hline & $s r x-97$ & $1.65 \pm 0.18(n=36)$ \\
\hline & odr-3 & $2.86 \pm 0.21(n=32)^{\star \star \star}$ \\
\hline \multirow{3}{*}{$\begin{array}{l}\mathrm{Cu}^{2+} \\
\quad(10 \mathrm{mM})\end{array}$} & WT & $1.13 \pm 0.03(n=30)$ \\
\hline & $s r x-97$ & $1.10 \pm 0.06(n=30)$ \\
\hline & odr-3 & $1.53 \pm 0.25(n=30)^{\star \star \star}$ \\
\hline \multirow{3}{*}{$\begin{array}{l}\text { Dihydrocaffeic acid } \\
\quad(100 \mathrm{~mm})\end{array}$} & WT & $1.85 \pm 0.11(n=30)$ \\
\hline & $s r x-97$ & $2.52 \pm 0.14(n=30)^{\star *}$ \\
\hline & odr-3 & $2.36 \pm 0.28(31)^{\star \star}$ \\
\hline \multirow{3}{*}{$\begin{array}{l}\text { Dihydrocaffeic acid } \\
\qquad(1 \mathrm{M})\end{array}$} & WT & $1.57 \pm 0.21(n=32)$ \\
\hline & $s r x-97$ & $1.68 \pm 0.32(n=31)$ \\
\hline & $o d r-3$ & $1.90 \pm 0.15(n=31)$ \\
\hline \multirow{3}{*}{$\begin{array}{l}\text { Acetic acid } \\
\qquad(0.1 \mathrm{M})\end{array}$} & WT & $3.88 \pm 0.15(n=31)$ \\
\hline & $s r x-97$ & $3.56 \pm 0.10(n=30)$ \\
\hline & $o d r-3$ & $5.77 \pm 0.20(n=30)^{\star \star \star}$ \\
\hline \multirow{3}{*}{$\begin{array}{l}\text { Acetic acid } \\
\quad(1 \mathrm{M})\end{array}$} & WT & $2.53 \pm 0.15(n=30)$ \\
\hline & srx-97 & $2.87 \pm 0.10(n=30)$ \\
\hline & $o d r-3$ & $4.58 \pm 0.10(n=30)^{\star \star \star}$ \\
\hline \multirow{3}{*}{$\begin{array}{l}\text { Quinine } \\
\qquad(10 \mathrm{~mm})\end{array}$} & WT & $2.49 \pm 0.20(n=35)$ \\
\hline & $s r x-97$ & $2.09 \pm 0.21(n=30)$ \\
\hline & $o d r-3$ & $2.89 \pm 0.16(n=32)$ \\
\hline \multirow{3}{*}{$\begin{array}{l}\text { Quinine } \\
\text { (1 } \mathrm{mm})\end{array}$} & WT & $10 \%(n=30)$ \\
\hline & srx-97 & $9 \%(n=30)$ \\
\hline & $o d r-3$ & $7 \%(n=30)$ \\
\hline \multirow[t]{3}{*}{ M13 buffer } & WT & $6 \%(n=30)$ \\
\hline & srx-97 & $4 \%(n=30)$ \\
\hline & odr-3 & $4 \%(n=30)$ \\
\hline
\end{tabular}

array that was undetectable with the fluorescence markers in these non-array (NA) lines. Further, in the WT background, the rescuing lines of SRX-97 behaved in a manner similar to WT control animals (Extended Data Fig. 3-1B). We also tested the previously described mCherrytagged SRX-97 line (Fig. $1 H$ ) in our rescue experiments and observed a partial rescue of the srx-97 mutant phenotype (Extended Data Fig. 3-1C, the rescue line showed no significant difference with respect to WT control animals or srx-97 mutants). This partial rescue could be by the mCherry tag hindering the function of SRX-97 or because of incomplete penetrance of expression from the arrays used. Thus far, our data suggest that the csGPCR SRX97 is responsible for sensing high concentrations of benzaldehyde.

\section{Ablation of ASH causes defects in benzaldehyde sensing}

We next analyzed the chemotaxis frequency of srx-97 mutants toward high concentrations of benzaldehyde (Nuttley et al., 2001). Here, we added the benzaldehyde $\left(10^{-1}\right)$ on a small sheet $(0.5-1 \mathrm{~cm}$ in diameter) of Parafilm so it would not be soaked in the media. We also excluded the addition of sodium azide on the control and test spots so as to allow the animals to move freely toward the control or test spots. After a 60-min incubation period, the animals were counted along each sector, and the
Table 5: Response of srx-97 mutants toward volatile chemicals

\begin{tabular}{lll}
\hline Volatile chemicals & Genotype & Cl index \\
\hline Diacetyl $\left(10^{-2}\right)$ & WT & $0.81(n=9)$ \\
& srx-97 & $0.73(n=9)$ \\
Diacetyl $\left(10^{-3}\right)$ & odr-3 & $0.50(n=9)^{\star *}$ \\
& WT & $0.85(n=9)$ \\
Isoamyl alcohol $\left(10^{-2}\right)$ & srx-97 & $0.84(n=6)$ \\
& odr-3 & $0.70(n=6)$ \\
& WT & $0.84(n=9)$ \\
Isoamyl alcohol $\left(10^{-3}\right)$ & srx-97 & $0.83(n=9)$ \\
& odr-3 & $0.78(n=9)$ \\
& WT & $0.94(n=6)$ \\
& srx-97 & $0.92(n=6)$ \\
& odr-3 & $0.87(n=6)$ \\
\hline
\end{tabular}

chemotaxis frequency was calculated by the formula indicated in Figure 4A. Again, the srx-97 mutants showed a significant increase in their attraction toward benzaldehyde (Fig. 4B). This defect was reduced by expressing SRX-97 under its endogenous promoter, suggesting that SRX-97 is responsible for sensing high concentrations of benzaldehyde and that the srx-97 phenotype may not be because of the initial attraction followed by repulsion behavior shown by the odr-3 mutants (Fig. 4B; Nuttley et al., 2001). However, a similar reduction of the defect was also observed in non-transgenic siblings that could be because of low expression of the rescuing array undetectable by fluorescence (Fig. 4B).

In order to further strengthen our data that SRX-97 was indeed acting in $\mathrm{ASH}$ to sense high concentrations of benzaldehyde, we ablated the ASH neurons in WT as well as in srx-97 mutant animals. We then tested the delay in response toward benzaldehyde in mock ablated and $\mathrm{ASH}$ ablated animals. Our data show that WT animals with ablated ASH neurons show a significant delay in their response to benzaldehyde when compared with the mockablated animals (Fig. 4C). Moreover, there was a significant difference in ASH ablated WT animals and mock ablated srx-97 mutant $C$. elegans, indicating that ASH may also have other receptors that allows detection of high concentrations of benzaldehyde. Animals where the ASH neurons were ablated in srx-97 mutants, behaved like WT animals that had undergone ASH neuron ablation, further indicating the function of SRX-97 in ASH neurons.

\section{Defects in sensory signaling appear to function downstream of srx-97}

The ASH neurons express multiple GPCR associated sensory molecules that are reported to be required for signal transduction (Roayaie et al., 1998; Hilliard et al., 2004, 2005). Among these, the G-protein subunit, GPC-1 that encodes the $\gamma$ subunit of GPCRs, shows a positive adaptive olfactory response toward benzaldehyde (Jansen et al., 2002; Yamada et al., 2009). We found that gpc-1 and srx-97 double mutants show a negative C.I. similar to what was seen with gpc-1 mutant animals (Fig. 5A). These data indicated that SRX-97 could be functioning through the G-protein signaling pathway. We next tested mutants in ODR-3, a $\mathrm{G} \alpha$ protein which is primarily 
A

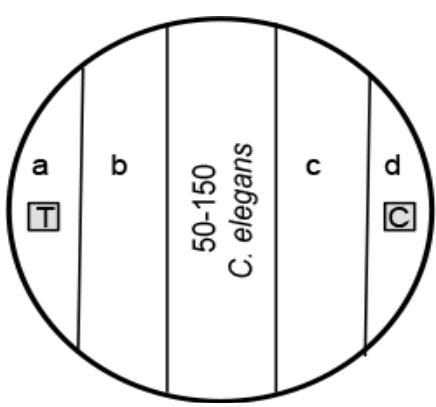

Chemotaxis frequency $=[$ No. of $C$. elegans in $(a+b)-$ No. of $C$. elegans in $(c+d)] /$ Total number of animals on plate

B

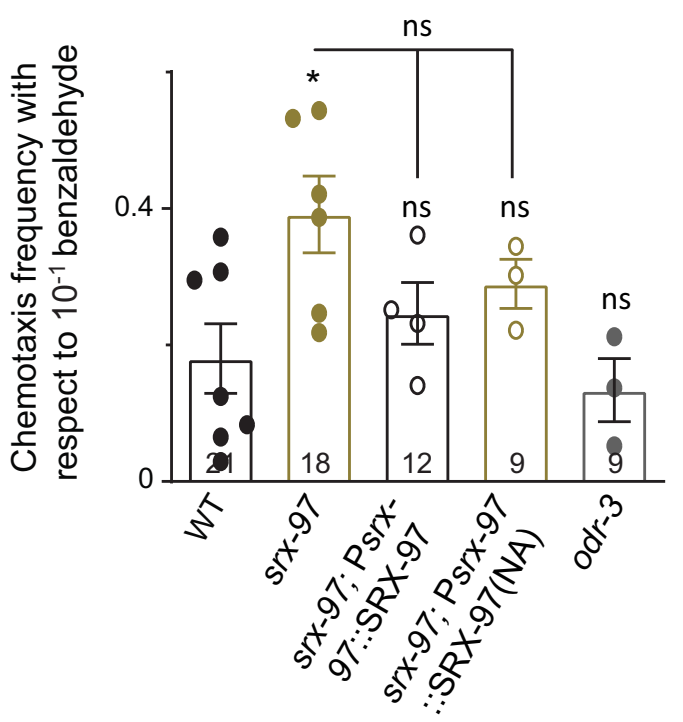

C

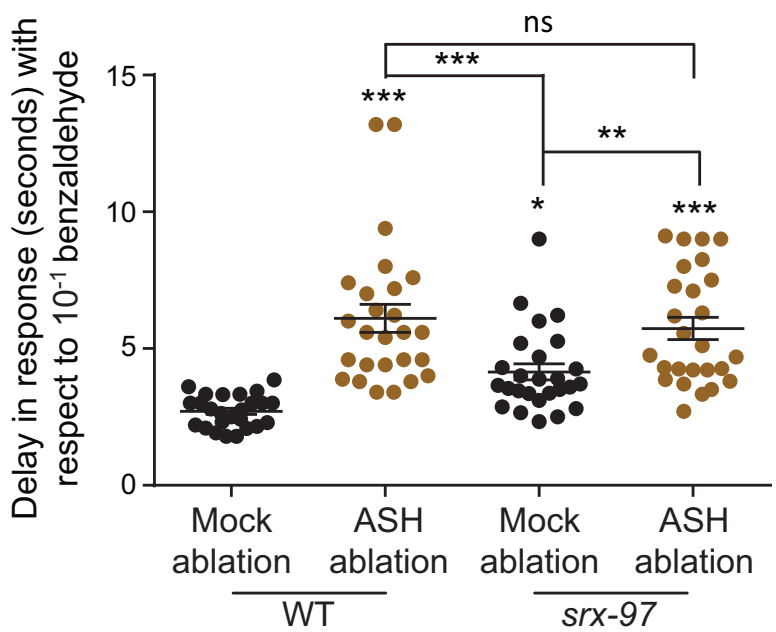

Figure 4. Ablation of ASH neurons shows defects toward chemosensation to benzaldehyde. $\boldsymbol{A}$, Illustration of the design of the plates used for analyzing the chemotaxis frequency of $C$. elegans along with the formula used for this calculation. Each sector (a-d) is $1 \mathrm{~cm}$ in width. $\boldsymbol{B}$, Graph of chemotaxis frequencies of WT, srx- required for sensory signal transduction and is involved in responses toward osmotic strength, high salt concentration, nose touch, and volatile chemicals (Roayaie et al., 1998; Hilliard et al., 2004, 2005; Zhang et al., 2016). Mutants in odr-3 have been reported to show defects in attraction toward low concentrations of benzaldehyde (1:200; Roayaie et al., 1998). In our assay, we found that odr-3 and srx-97; odr-3 double mutants showed negative chemotaxis indices toward high concentration of benzaldehyde, similar to what was seen with gpc-1 mutants (Fig. 5B). These data further suggest that SRX-97 could be involved in chemotactic function through the GPCR pathway.

The AWC neurons sense low concentration of benzaldehyde through odr-3 signaling (Bargmann et al., 1993). To gain more insight into the function of AWC neurons in sensing benzaldehyde, we used a line where AWC is ablated [AWC(-); Beverly et al., 2011]. We found that the loss of AWC neurons made the $C$. elegans aversive toward benzaldehyde (Fig. 5B). Further loss of srx-97 in the AWC $(-)$ background did not appear to affect the AWC(-) phenotype (Fig. 5B). These data suggest that AWC is the primary sensory neuron that shows attraction to diffused (in our assay) or low concentrations of benzaldehyde. Further, the ASH neurons could act as secondary sets of neurons that are responsive to high benzaldehyde concentrations as shown previously for IAA (Yoshida et al., 2012). We also found that the loss of odr-3 in the AWC(-) animals appeared to significantly suppress the AWC ablation defects with respect to chemotaxis toward benzaldehyde (Fig. 5B). Studies have shown that ODR-3 is expressed in ASH, AWA, AWB and AWC neurons (Roayaie et al., 1998). Hence it is possible that ODR-3 might be affecting chemotaxis toward benzaldehyde through neurons other than AWC.

We next analyzed the molecules that may be functioning downstream of the SRX-97 GPCR. The ASH neurons express multiple channel proteins that get activated through GPCRs and are involved in regulating different behavioral outputs (Sengupta, 2007). One such molecule, OSM-9, is a member of the vanilloid subfamily of TRP channel proteins that regulates avoidance behaviors to osmotic strength, nose touch and undiluted benzaldehyde in the ASH neurons (Colbert et al., 1997; Murayama and Maruyama, 2013; Zou et al., 2017). We found that

\section{continued}

97, the srx-97 rescue line and a control odr-3 mutant line to a high concentration of benzaldehyde. The assay was performed in triplicate over multiple days with each dot indicating an assay done in triplicate. The numbers at the base of each plot indicate the number of times the experiment was performed with each genotype. C, Graph plotting the delay in response of animals toward a high benzaldehyde concentration. The animals used in this experiment have undergone mock ablation or ASH ablation in WT or srx-97 mutant backgrounds. Each dot indicates a response from a single animal. Approximately 25 mock ablated animals and ASH ablated animals in WT and srx-97 mutant background were analyzed for this experiment over multiple days. The error bars represent SEM, and statistical significance is represented as "ns" for not significant; ${ }^{*} p<0.05,{ }^{\star \star} p<0.01,{ }^{* \star *} p<0.001$. 
A

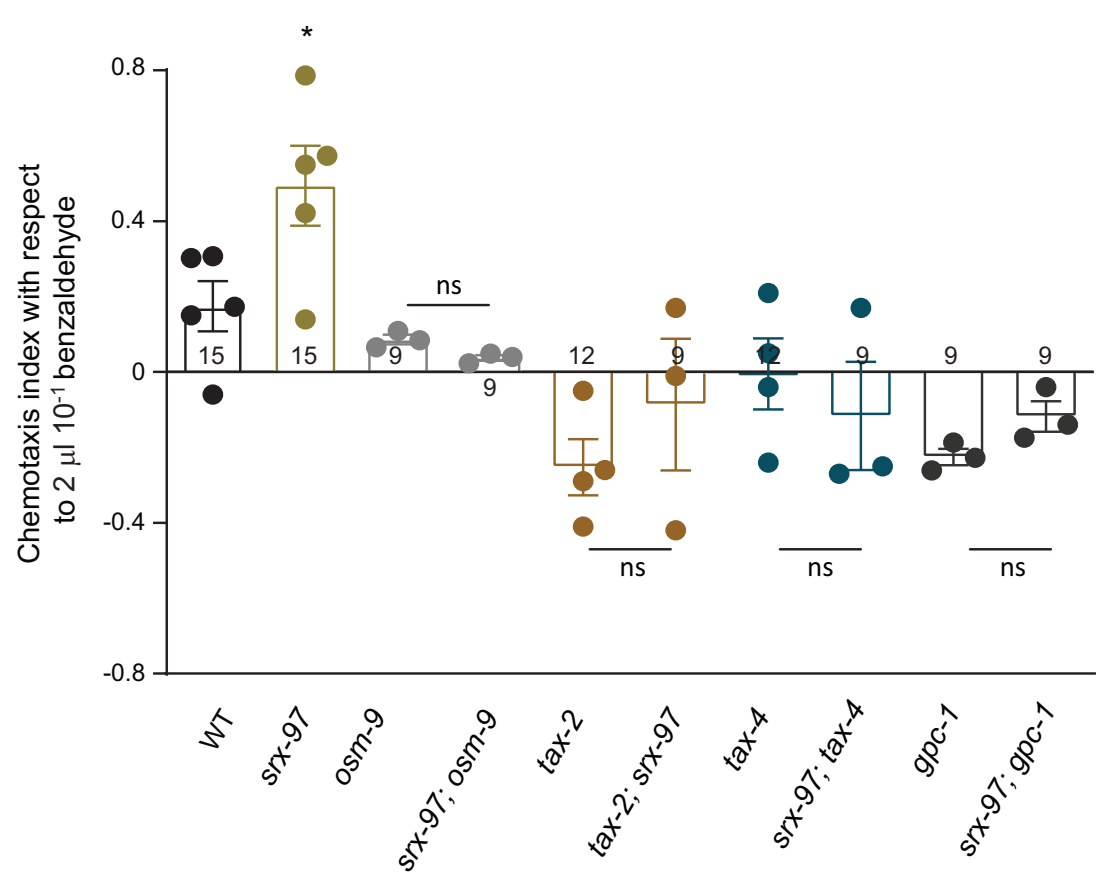

B

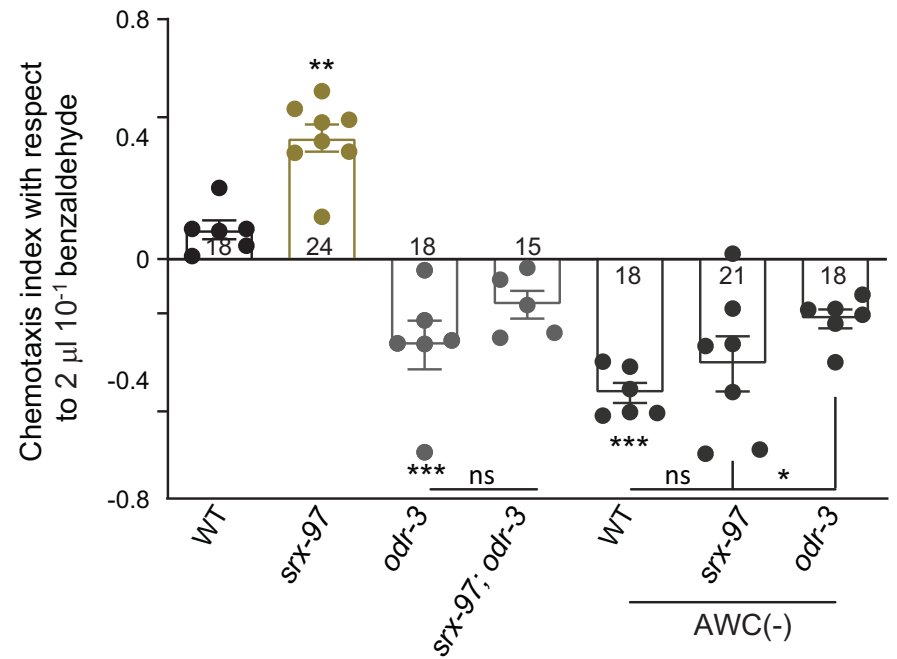

Figure 5. The srx-97 mutant phenotype is suppressed by other signaling mutants that appear to function downstream of SRX-97. $\boldsymbol{A}$, Chemotaxis indices with respect to high concentration of benzaldehyde in WT, srx-97, osm-9, tax-2, tax-4, and gpc-1 mutants along with analysis of each mutant in the $s r x-97$ background. $\boldsymbol{B}$, Chemotaxis indices with respect to high concentration of benzaldehyde in WT, srx-97, odr-3, and srx-97; odr-3 mutants. This graph also indicates chemotaxis indices with respect to benzaldehyde on ablation of the AWC neuron [AWC(-)] in WT, srx-97, and odr-3 mutants. The assays were performed in triplicate over multiple days. Each dot in both plots $\boldsymbol{A}, \boldsymbol{B}$ indicates an assay done in triplicate. The numbers at the base of each graph in $\boldsymbol{A}, \boldsymbol{B}$ indicate the total number of times the experiment was performed with each genotype. The error bars represent SEM, and statistical significance is represented as "ns" for not significant; ${ }^{\star} p<0.05$, ${ }^{\star *} p<0.01,{ }^{\star \star *} p<0.001$.

osm-9 mutants showed a phenotype similar to that seen in WT animals when tested for a high concentration of benzaldehyde (Fig. 5A). The cyclic nucleotide-gated channel proteins, TAX-2 and TAX-4 are responsible for the detection of volatile chemicals like benzaldehyde by AWC and other amphid neurons, although the source of activating cGMP is still unknown (Coburn and Bargmann, 1996; Komatsu et al., 1996; Zagotta and Siegelbaum, 1996). These mutants were tested for defects in chemotaxis to benzaldehyde and showed a negative C.I. toward a high concentration of benzaldehyde (Fig. 5A). All three mutants, osm-9, tax-2, and tax4 , completely suppressed the increased chemotaxis behavior seen in srx-97 mutant animals. The suppression of the srx-97 mutant phenotype by these downstream molecules indicates that either the SRX-97 GPCR acts redundantly to sense the high concentrations of benzaldehyde by activating pathways different from the ones tested, or OSM-9, TAX-2, and TAX-4 function to detect both high and low 
concentrations of benzaldehyde and SRX-97 functions through the canonical G-protein pathway to elicit responses to high concentrations of benzaldehyde.

\section{Discussion}

In this study, we have characterized the expression and function of the GPCR, SRX-97. From our expression studies, it is clear that SRX-97 shows expression in the ASH and PHB neurons. Moreover, the chemotaxis experiments reveal that the GPCR SRX-97 senses high concentrations of benzaldehyde. Our data indicate that in comparison with WT animals, srx-97 null mutant animals show increased attraction toward high concentrations of benzaldehyde $\left(10^{-1}\right)$. We also show that SRX-97::mCherry driven by its native promoter shows localization along the ciliary tip of the ASH neurons. Since the cilia are the compartment where signal sensation and transduction occur, the localization of SRX-97 at the cilium tips suggests its role in sensory perception or transduction of sensory sig$\mathrm{nal} / \mathrm{s}$. These results suggest that SRX-97 expressed in the ASH neurons is responsible for detecting benzaldehyde from its surroundings.

Other than SRX-97, ASH neurons also express different sets of GPCRs, which sense benzaldehyde (Aoki et al., 2011; Taniguchi et al., 2014; Vidal et al., 2018). For example, DCAR-1 is expressed in ASH neurons and is involved in sensing undiluted benzaldehyde (Aoki et al., 2011). Multiple reports propose that there is a "tuning curve" for the olfactory neurons through which some olfactory receptors exhibit noticeable sensitivity (threshold) for some odorants; some neurons are activated by receptors only at low odorant concentrations, while other neurons and receptors are activated at high concentrations of odorants (Firestein, 2001; Spehr and Munger, 2009). Since the srx97 mutant animals show reduced but not completely abolished response toward high concentrations $\left(10^{-1}\right)$ of benzaldehyde (Figs. 3G, 4C), it is possible that SRX-97 acts as a constituent of a receptor complex on the ASH neurons allowing for detection of benzaldehyde at high or undiluted concentrations but not at low concentrations. On the contrary, low concentrations of benzaldehyde are is sensed by the AWC neurons (Bargmann et al., 1993; Leinwand et al., 2015). The WT like chemotaxis response of $s r x-97$ mutants toward undiluted and low concentration of benzaldehyde may suggest that animals sense their surrounding by activating different receptors using the corresponding neurons in a concentration-dependent manner and this, in turn, leads to appropriate behavioral responses.

GPCRs signal through heteromeric G-proteins signaling cascades and transduce signals from the environment through intracellular mediators that play a vital role in triggering behavior. The ASH and other amphid neurons express the $\mathrm{G} \alpha$ protein ODR-3 as well as OSM-9, a TRPV protein that is involved in the sensation of various stimuli including olfaction (Bargmann et al., 1993; Troemel et al., 1997; Roayaie et al., 1998; Hilliard et al., 2004). The amphid AWC neurons act as primary olfactory neurons involved in sensing low concentrations of benzaldehyde (Leinwand et al., 2015), while the ASH neurons are required for sensing undiluted benzaldehyde (Troemel et al., 1995; Colbert et al., 1997; Tobin et al., 2002). In our chemotaxis assay ( $90 \mathrm{~min})$, we found that $\mathrm{srx}-97$ mutants presented chemotaxis defects toward high concentrations of benzaldehyde; in these assays the animals were placed $3 \mathrm{~cm}$ away from the source of benzaldehyde and hence not at short range from the source (Troemel et al., 1995). Previous work has shown that the distance or diffusion gradient of a test chemical may activate primary sensory neurons like AWA and AWC (Yoshida et al., 2012; Taniguchi et al., 2014; Leinwand et al., 2015). Our work also indicates that defects in the downstream signaling molecules in these neurons could affect the repulsion of the animals from the source.

The defects seen in the srx-97 mutation toward DHCA is similar to the previously reported GPCR/dcar-1 mutants expressed in ASH neurons (Aoki et al., 2011). DCAR-1 functions through odr-3 and osm-9 signaling pathways to elicit its response toward DHCA (Aoki et al., 2011). However, the downstream molecules for the signaling pathway remain unidentified. We also see that $s r x-97$ mutant animals show defects toward high osmolarity as seen in experiments using $2 \mathrm{~m}$ glycerol. Although in our battery of tests we saw small but significant defects in srx-97 mutants toward $2 \mathrm{~m}$ glycerol and $100 \mathrm{~mm}$ DHCA, we do not know the mechanisms underlying these defects in the mutant animals. It will be interesting in future to understand how single GPCRs like SRX-97 and their associated proteins or downstream molecules are responsible for these different aspects of chemosensation in C. elegans.

Our results propose that there could be alternative pathways for signal transduction in ASH neurons through GPCRs like SRX-97. To our knowledge, downstream signaling molecules, the loss of which causes attraction to an undiluted or high concentration of benzaldehyde through the ASH neurons have not yet been identified. The $C$. elegans genome encodes for $21 \mathrm{G} \alpha$, two $\mathrm{G} \beta$, and two $\mathrm{G}_{\gamma}$ genes (Jansen et al., 1999; Cuppen et al., 2003). Out of these, $11 \mathrm{G} \alpha$ proteins are expressed in the ASH neurons (Bastiani and Mendel, 2006). Our results suggest that the ASH neurons are involved in aversion to undiluted or high concentrations of benzaldehyde through multiple or redundant chemosensory pathways involved in the signaling through GPCRs like SRX-97.

In conclusion, our results bring out the possibility that SRX-97 is a key mediator in chemotaxis toward high concentrations of benzaldehyde in the chemosensory system of $C$. elegans. However, the downstream signaling components still need to be identified, to provide more details into the functioning of SRX-97 in the ASH neurons. These investigations may offer insights into the nature of signal transduction in ASH neurons and their physiological role in concentration-dependent avoidance responses.

\section{References}

Aoki R, Yagami T, Sasakura H, Ogura KI, Kajihara Y, Ibi M, Miyamae T, Nakamura F, Asakura T, Kanai Y, Misu Y, lino Y, Ezcurra M, Schafer WR, Mori I, Goshima Y (2011) A seven-transmembrane receptor that mediates avoidance response to dihydrocaffeic acid, a 
water-soluble repellent in Caenorhabditis elegans. J Neurosci 31:16603-16610.

Avery L, Horvitz HR (1987) A cell that dies during wild-type C. elegans development can function as a neuron in a ced-3 mutant. Cell 51:1071-1078.

Avery L, Horvitz HR (1989) Pharyngeal pumping continues after laser killing of the pharyngeal nervous system of $C$. elegans. Neuron 3:473-485.

Babu K, Hu Z, Chien SC, Garriga G, Kaplan JM (2011) The immunoglobulin super family protein RIG-3 prevents synaptic potentiation and regulates Wnt signaling. Neuron 71:103-116.

Bargmann Cl (2006) Chemosensation in C. elegans, pp 1-29. WormBook.

Bargmann Cl, Horvitz HR (1991) Chemosensory neurons with overlapping functions direct chemotaxis to multiple chemicals in C. elegans. Neuron 7:729-742.

Bargmann Cl, Hartwieg E, Horvitz HR (1993) Odorant-selective genes and neurons mediate olfaction in C. elegans. Cell 74:515527.

Bastiani C, Mendel J (2006) Heterotrimeric G proteins in C. elegans, pp 1-25. WormBook.

Basu A, Dey S, Puri D, Das Saha N, Sabharwal V, Thyagarajan P, Srivastava P, Koushika SP, Ghosh-Roy A (2017) let-7 miRNA controls CED-7 homotypic adhesion and EFF-1-mediated axonal selffusion to restore touch sensation following injury. Proc Natl Acad Sci USA 114:E10206-E10215.

Beverly M, Anbil S, Sengupta P (2011) Degeneracy and neuromodulation among thermosensory neurons contribute to robust thermosensory behaviors in Caenorhabditis elegans. J Neurosci 31:11718-11727.

Bhardwaj A, Thapliyal S, Dahiya Y, Babu K (2018) FLP-18 functions through the G-protein-coupled receptors NPR-1 and NPR-4 to modulate reversal length in Caenorhabditis elegans. J Neurosci 38:4641-4654.

Bhardwaj A, Pandey P, Babu K (2020) Control of locomotory behavior of Caenorhabditis elegans by the immunoglobulin superfamily protein RIG-3. Genetics 214:135-145.

Brenner S (1974) The genetics of Caenorhabditis elegans. Genetics 77:71-94.

Chao MY, Komatsu H, Fukuto HS, Dionne HM, Hart AC (2004) Feeding status and serotonin rapidly and reversibly modulate a Caenorhabditis elegans chemosensory circuit. Proc Natl Acad Sci USA 101:15512-15517.

Coburn CM, Bargmann Cl (1996) A putative cyclic nucleotide-gated channel is required for sensory development and function in C. elegans. Neuron 17:695-706.

Colbert HA, Smith TL, Bargmann CI (1997) OSM-9, a novel protein with structural similarity to channels, is required for olfaction, mechanosensation, and olfactory adaptation in Caenorhabditis elegans. J Neurosci 17:8259-8269.

Cuppen E, van der Linden AM, Jansen G, Plasterk RH (2003) Proteins interacting with Caenorhabditis elegans Galpha subunits. Comp Funct Genomics 4:479-491.

Dahiya Y, Rose S, Thapliyal S, Bhardwaj S, Prasad M, Babu K (2019) Differential regulation of innate and learned behavior by Creb1/ $\mathrm{CRH}-1$ in Caenorhabditis elegans. J Neurosci 39:7934-7946.

Dickinson DJ, Goldstein B (2016) CRISPR-based methods for Caenorhabditis elegans genome engineering. Genetics 202:885901.

Erlandson SC, McMahon C, Kruse AC (2018) Structural basis for G protein-coupled receptor signaling. Annu Rev Biophys 47:1-18.

Firestein S (2001) How the olfactory system makes sense of scents. Nature 413:211-218.

Fredriksson R, Lagerström MC, Lundin LG, Schiöth HB (2003) The G-protein-coupled receptors in the human genome form five main families. Phylogenetic analysis, paralogon groups, and fingerprints. Mol Pharmacol 63:1256-1272.

Gray JM, Hill JJ, Bargmann Cl (2005) A circuit for navigation in Caenorhabditis elegans. Proc Natl Acad Sci USA 102:3184-3191.
Hart AC, Kass J, Shapiro JE, Kaplan JM (1999) Distinct signaling pathways mediate touch and osmosensory responses in a polymodal sensory neuron. J Neurosci 19:1952-1958.

Hilliard MA, Bargmann Cl, Bazzicalupo P (2002) C. elegans responds to chemical repellents by integrating sensory inputs from the head and the tail. Curr Biol 12:730-734.

Hilliard MA, Bergamasco C, Arbucci S, Plasterk RH, Bazzicalupo P (2004) Worms taste bitter: ASH neurons, QUI-1, GPA-3 and ODR3 mediate quinine avoidance in Caenorhabditis elegans. EMBO J 23:1101-1111.

Hilliard MA, Apicella AJ, Kerr R, Suzuki H, Bazzicalupo P, Schafer WR (2005) In vivo imaging of $C$. elegans ASH neurons: cellular response and adaptation to chemical repellents. EMBO J 24:63-72.

Hsu PD, Scott DA, Weinstein JA, Ran FA, Konermann S, Agarwala V, Li Y, Fine EJ, Wu X, Shalem O, Cradick TJ, Marraffini LA, Bao G, Zhang F (2013) DNA targeting specificity of RNA-guided Cas9 nucleases. Nat Biotechnol 31:827-832.

Jansen G, Thijssen KL, Werner P, van der Horst M, Hazendonk E, Plasterk RH (1999) The complete family of genes encoding G proteins of Caenorhabditis elegans. Nat Genet 21:414-419.

Jansen G, Weinkove D, Plasterk RH (2002) The G-protein gamma subunit gpc-1 of the nematode $C$. elegans is involved in taste adaptation. EMBO J 21:986-994.

Kaplan JM, Horvitz HR (1993) A dual mechanosensory and chemosensory neuron in Caenorhabditis elegans. Proc Natl Acad Sci USA 90:2227-2231.

Katritch V, Cherezov V, Stevens RC (2013) Structure-function of the $G$ protein-coupled receptor superfamily. Annu Rev Pharmacol Toxicol 53:531-556.

Komatsu H, Mori I, Rhee JS, Akaike N, Ohshima Y (1996) Mutations in a cyclic nucleotide-gated channel lead to abnormal thermosensation and chemosensation in C. elegans. Neuron 17:707-718.

Lagerstrom MC, Schioth HB (2008) Structural diversity of G proteincoupled receptors and significance for drug discovery. Nat Rev Drug Discov 7:339-357.

Leinwand SG, Yang CJ, Bazopoulou D, Chronis N, Srinivasan J, Chalasani SH (2015) Circuit mechanisms encoding odors and driving aging-associated behavioral declines in Caenorhabditis elegans. Elife 4:e10181.

Mahoney TR, Luo S, Nonet ML (2006) Analysis of synaptic transmission in Caenorhabditis elegans using an aldicarb-sensitivity assay. Nat Protoc 1:1772-1777.

Margie O, Palmer C, Chin-Sang I (2013) C. elegans chemotaxis assay. J Vis Exp. Advance online publication. Retrieved April 27, 2013. doi: $10.3791 / 50069$.

Maricq AV, Peckol E, Driscoll M, Bargmann Cl (1995) Mechanosensory signalling in $C$. elegans mediated by the GLR-1 glutamate receptor. Nature 378:78-81.

Mello C, Fire A (1995) DNA transformation. Methods Cell Biol 48:451-482.

Mello CC, Kramer JM, Stinchcomb D, Ambros V (1991) Efficient gene transfer in $C$. elegans: extrachromosomal maintenance and integration of transforming sequences. EMBO J 10:3959-3970.

Murayama T, Maruyama IN (2013) Decision making in C. elegans chemotaxis to alkaline $\mathrm{pH}$ : competition between two sensory neurons, ASEL and ASH. Commun Integr Biol 6:e26633.

Nuttley WM, Harbinder S, van der Kooy D (2001) Regulation of distinct attractive and aversive mechanisms mediating benzaldehyde chemotaxis in Caenorhabditis elegans. Learn Mem 8:170-181.

Pokala N, Liu Q, Gordus A, Bargmann Cl (2014) Inducible and titratable silencing of Caenorhabditis elegans neurons in vivo with histamine-gated chloride channels. Proc Natl Acad Sci USA 111:27702775.

Roayaie K, Crump JG, Sagasti A, Bargmann Cl (1998) The G alpha protein ODR-3 mediates olfactory and nociceptive function and controls cilium morphogenesis in $C$. elegans olfactory neurons. Neuron 20:55-67.

Robertson HM, Thomas JH (2006) The putative chemoreceptor families of C. elegans, pp 1-12. WormBook. 
Sambrook JF, Russell DW (2001) Molecular cloning: a laboratory manual. Cold Spring Harbor: Cold Spring Harbor Laboratory Press.

Sengupta $P$ (2007) Generation and modulation of chemosensory behaviors in C. elegans. Pflugers Arch 454:721-734.

Sengupta $\mathrm{P}$, Chou JH, Bargmann $\mathrm{Cl}$ (1996) odr-10 encodes a seven transmembrane domain olfactory receptor required for responses to the odorant diacetyl. Cell 84:899-909.

Spehr M, Munger SD (2009) Olfactory receptors: G protein-coupled receptors and beyond. J Neurochem 109:1570-1583.

Taniguchi G, Uozumi T, Kiriyama K, Kamizaki T, Hirotsu T (2014) Screening of odor-receptor pairs in Caenorhabditis elegans reveals different receptors for high and low odor concentrations. Sci Signal 7:ra39.

Tobin DM, Madsen DM, Kahn-Kirby A, Peckol EL, Moulder G, Barstead R, Maricq AV, Bargmann Cl (2002) Combinatorial expression of TRPV channel proteins defines their sensory functions and subcellular localization in C. elegans neurons. Neuron 35:307318.

Troemel ER, Chou JH, Dwyer ND, Colbert HA, Bargmann Cl (1995) Divergent seven transmembrane receptors are candidate chemosensory receptors in C. elegans. Cell 83:207-218.

Troemel ER, Kimmel BE, Bargmann Cl (1997) Reprogramming chemotaxis responses: sensory neurons define olfactory preferences in C. elegans. Cell 91:161-169.
Vidal B, Aghayeva U, Sun H, Wang C, Glenwinkel L, Bayer EA, Hobert O (2018) An atlas of Caenorhabditis elegans chemoreceptor expression. PLoS Biol 16:e2004218.

Walker DS, Vázquez-Manrique RP, Gower NJ, Gregory E, Schafer WR, Baylis HA (2009) Inositol 1,4,5-trisphosphate signalling regulates the avoidance response to nose touch in Caenorhabditis elegans. PLoS Genet 5:e1000636.

Yamada K, Hirotsu T, Matsuki M, Kunitomo H, lino Y (2009) GPC-1, a $G$ protein gamma-subunit, regulates olfactory adaptation in Caenorhabditis elegans. Genetics 181:1347-1357.

Yoshida K, Hirotsu T, Tagawa T, Oda S, Wakabayashi T, lino Y, Ishihara T (2012) Odour concentration-dependent olfactory preference change in C. elegans. Nat Commun 3:739.

Zagotta WN, Siegelbaum SA (1996) Structure and function of cyclic nucleotide-gated channels. Annu Rev Neurosci 19:235-263.

Zhang C, Zhao N, Chen Y, Zhang D, Yan J, Zou W, Zhang K, Huang $X$ (2016) The signaling pathway of Caenorhabditis elegans mediates chemotaxis response to the attractant 2-heptanone in a trojan horse-like pathogenesis. J Biol Chem 291:23618-23627.

Zheng M, Cao P, Yang J, Xu XZ, Feng Z (2012) Calcium imaging of multiple neurons in freely behaving $C$. elegans. J Neurosci Methods 206:78-82.

Zou W, Cheng H, Li S, Yue X, Xue Y, Chen S, Kang L (2017) Polymodal responses in $C$. elegans phasmid neurons rely on multiple intracellular and intercellular signaling pathways. Sci Rep 7:42295. 